\title{
MODELADO PARA LA EVALUACIÓN DE ALTERNATIVAS DE POLÍTICAS PÚBLICAS EN LA GESTIÓN DEL AGUA EN LA REGIÓN HÍDRICA BOGOTÁ - COLOMBIA
}

\author{
A model for the evaluation of public policy alternatives in Bogota's water managment
}

\author{
Andrés Chavarro \\ Politécnico Grancolombiano \\ achavarr@poligran.edu.co \\ Mónica Castañeda \\ Universidad Jorge Tadeo Lozano \\ monica.castanedar@utadeo.edu.co \\ Sebastián Zapata \\ Universidad Jorge Tadeo Lozano \\ Sebatian.zapatar@utadeo.edu.co \\ Isaac Dyner \\ Universidad Jorge Tadeo Lozano \\ Isaac.dynerr@utadeo.edu.co
}

Resumen

En la región hídrica de Bogotá se genera el 17\% del PIB de Colombia y alberga el 20\% de la población del país (DANE, 2017). Durante las últimas dos décadas, los informes públicos sobre el estado del recurso hídrico en la cuenca del río Bogotá establecen que el índice de Vulnerabilidad Hídrica por desabastecimiento alcanza la calificación muy alto (IDEAM 2000, 2010, 2015; Huitaca CAR, 2018) mientras que estudios sobre el impacto combinado del cambio climático y aumento de la población aumentarían la demanda hasta 50\% en 2050 (Buytaert W. \&., 2012). En dicho contexto se precisa identificar las condiciones para garantizar que el suministro de agua en la región sea sostenible en el largo plazo. En orden a lograr el objetivo se usó el modelado de dinámica de sistemas que hasta el momento no se ha usado para el caso de estudio seleccionado. Se encontró que si los rezagos en la ampliación de la oferta se sitúan entre los 5 y 9 años y las políticas de gestión de la demanda se aplican suficientemente, contando con un crecimiento tendencial de la demanda y sin la influencia del cambio y la variabilidad climática, no se avizora desabastecimiento.

Palabras clave:

Oferta de agua; Dinámica de sistema; cuenca del Rio Bogotá; Escasez de agua 


\section{INTRODUCCIÓN}

A principios de 2018, en Ciudad del Cabo, se presentó una crisis por falta de agua para consumo humano que forzó a los gobernantes a plantear a sus ciudadanos la posibilidad que no hubiera agua disponible para el consumo a partir del 22 abril (día cero). Mientras que esta ciudad portuaria y turística sufre la peor sequía del siglo, su ciclo de lluvias se movió de marzo a mayo y el promedio de precipitaciones ha disminuido, su población se ha incrementado $50 \%$ en los últimos 20 años, el consumo per cápita por día se ha reducido a 87 litros y la capacidad de las represas creció 15\% desde 1995 (Redacción BBC Mundo, 2018).

Recientemente, casos similares se registran a lo largo del mundo. San Pablo y Ciudad de México son solo dos ejemplos. En San Pablo, en 2014, la represa Cantareira llegó a su límite histórico de 3,5\%. Empezaron las restricciones al consumo y se llegó a contemplar la posibilidad que el agua se acabara a julio de 2015, si las obras de interconexión de represas no se llevaban a cabo (Martín, 2015). En ciudad de México la fragilidad del sistema de abastecimiento amenaza con llegar a una crisis hídrica en 10 años debido a la sobreexplotación de los acuíferos, fugas del sistema de tuberías y el no aprovechamiento de las aguas residuales (Silva \& Plascencia, 2016).

Mientras tanto estudios que se centran en países andinos como Perú, Ecuador y Colombia sugieren que el efecto combinado del cambio climático y el crecimiento demográfico podría incrementar la demanda hasta 50\% para 2050 (Buytaert W. \&., 2012) (for Bogotá and Quito), (Flóres-López, Galaitsi, Escobar \& Purkey, 2016)

De acuerdo con la nota técnica Aqueduct 3.0 (HOFSTE, y otros, 2019) alrededor de 2600 millones de personas, viven en países con una situación de estrés hídrico "extremadamente alto". La región del mundo con mayor estrés hídrico es el medio oriente y le sigue el subcontinente de la India que muestra graves problemas con el uso y gestión del agua. Para el caso de América latina los países con mayor riesgo de escasez son Chile (donde 10 de las 16 regiones del país sufren escasez extrema de agua) y México que tiene un sistema de agua muy frágil, y que si no toma medidas, llegará a una situación tan grave como la de la India.

Las ciudades con desafíos importantes a la hora de garantizar el suministro adecuado en términos de cantidad, calidad y regularidad de agua se caracterizan por tener dificultades en la gestión del territorio (van den Brandeler, Gupta, \& Hordijk, 2018), un diseño institucional que no permite canalizar el uso y conservación del recurso en una perspectiva de largo plazo (Räsänen, y otros, 2017), el precio del agua es muy bajo y no sirve como instrumento efectivo de política ambiental, (Rudas, 2012), una insuficiente infraestructura que garantice el suministro (Varis, Biswas, Tortajada, \& Lundqvist, 2006) (Hossain \& Bahauddin, 2013) (Costa, Burlando, \& Priadi, 2016) al mismo tiempo que una demanda creciente y mayor exposición a los efectos del cambio climático.

En el centro de Colombia, sobre el costado oriental de la cordillera de Los Andes está la cuenca del río Bogotá. Allí, se genera el $26 \%$ del PIB y vive el 20\% de la población de la nación (DANE, 2020). De acuerdo con el más reciente Estudio Nacional del Agua (IDEAM, 2019), en un año con un nivel promedio precipitaciones, la cuenca del río Bogotá presenta un índice de vulnerabilidad hídrica al desabastecimiento "muy alto". En gran medida podría explicarse por dos grandes razones: 
1) una inadecuada gestión del territorio, es decir, crecimiento urbano sin planificación de largo plazo, (Ricardo, 2014), conurbación desordenada (Osorio, 2008); (Motta, 2009); (Gaitan, 2010) presión por expansión urbana (Instituto Alexander von Humboldt, 2016), soluciones de vivienda en zonas de riesgo de inundación (Rojas, De Meulder, \& Shannon, 2015), destrucción de ecosistemas (Pérez-Hernández, 1998).

2) Fragilidad en el diseño institucional y de los instrumentos de gestión del recurso, esto es, los planes de ordenamiento de cuenca son descriptivos y no establecen restricciones al uso de la tierra, los procedimientos de concesiones son centralizados largos y costosos para los pequeños usuarios de agua y los precios del agua, instrumento que puede ser efectivo en el desincentivo al consumo, son muy bajos (Guhl-Nanneti, 2013), (Rudas, 2012).

El problema del desabastecimiento de agua para la región ha sido estudiado desde diferentes perspectivas: modelos de cambio climático, modelos hidrológicos y modelos de flujos de materiales. En el primer caso Buytaert \& De Bièvre (2012) estiman que el efecto combinado de aumento de la temperatura promedio y un aumento de la población podría aumentar la demanda hasta en un 50\% en 2050. Entidades públicas como el instituto de estudios ambientales y el gobierno de Bogotá, encuentran que "hay indicios de cambio climático en la región con aumentos de temperatura de $2^{\circ} \mathrm{C}$ " (IDEAM; PNUD; Alcaldía de Bogotá; Gobernación de Cundinamarca; CAR; Corpoguavio; Instituto Alexander von Humboldt; Parques Nacionales Naturales de Colombia; MADS; DNP, 2014, pág. 8). Desde la perspectiva de modelos hidrológicos, los Estudios Nacionales del agua desde 2000 hasta 2019 muestran consistentemente que el índice de vulnerabilidad al desabastecimiento (IVH) para la cuenca del río Bogotá está en la categoría muy alto.

Por último, desde la perspectiva de flujo de materiales. Díaz (2011) cuantifica el balance global de materia (alimentos y agua) y energía para Bogotá entre 1980 y 2010. Los resultados muestran que en el periodo analizado las entradas de agua se mantuvieron constantes por persona alrededor de los $16,5 \mathrm{~m} 3 / \mathrm{s}$ cada año y las salidas (pérdidas, vertimientos y escorrentía no aprovechada), salvo este último, los demás crecieron de forma importante indicando que la infraestructura disponible dilapida recurso hídrico afectando la disponibilidad. Por su parte, Ivanova (2013) calculó la huella hídrica total para la ciudad de Bogotá para 2008 lo que le permitió concluir que el consumo urbano está controlado pero los vertimientos son cada vez mayores con lo cual de todas formas se incrementa la presión sobre el recurso.

Tanto los modelos de cambio climático como los modelos hidrológicos son herramientas importantes en la planeación del recurso porque hacen análisis integrados (oferta y demanda) de la situación y perspectivas del posible desabastecimiento para la región hídrica de Bogotá. No obstante, no permiten la inclusión y evaluación de alternativas de gestión del problema de suerte que se procure un suministro adecuado en cantidad, calidad y continuidad para esta región clave del país. Así mismo, los modelos de flujos de materiales permiten análisis sistémicos del problema de desabastecimiento al incluir la dimensión de alimentos y energía como variables que afectan y son afectados por la disponibilidad de agua, pero no logran incorporar con suficiente detalle relaciones entre los elementos del sistema de abastecimiento en el contexto de la cuenca y cómo el cambio de estas en el tiempo, inciden en la disponibilidad futura del recurso.

Por ello, se requiere un modelado sistémico que permita identificar las condiciones de largo plazo que garanticen el suministro adecuado de agua para la región hídrica de Bogotá. 


\section{Planteamiento del problema}

Colombia es un país que se puede considerar como uno de los mejor dotados en cuanto a recurso hídrico en comparación con el resto del mundo. Ello se debe principalmente a su ubicación. El país se encuentra en la zona de convergencia intertropical limitada al norte y al occidente por dos océanos, atravesada por la cordillera de Los Andes y al sur hace parte de la gran región amazónica caracterizada por su geografía selvática.

La forma en que estos tres elementos confluyen para generar riqueza hídrica es la siguiente: la cordillera de Los Andes en su vertiente oriental recibe la humedad generada por la evapotranspiración desde la Amazonía que al chocar con las montañas da origen a copiosas lluvias mientras que, en la vertiente occidental, los valores de precipitación se explican por la corriente marina fría Humbolt originada en la Antártida. En la zona de precipitación de Los Andes ecuatorianos y colombianos la temperatura del mar aumenta, lo que permite que haya grandes volúmenes de lluvia (Guhl-Nanneti, 2013).

Por su parte, la demanda de agua se concentra en nueve usos: sector agropecuario (43,1\%), energía (24,3\%), pecuario (8,2\%), piscícola ( $8,1 \%)$, el consumo doméstico ( $7,4 \%$ ), industrial (2,9\%), minero(1,8\%), hidrocarburos (1,5\%) y servicios(1,5\%) para un consumo agregado de $37.308,3 \mathrm{Mm}^{3}$ a 2016 (IDEAM, 2019).

El mismo informe reporta en términos de balance hídrico que la zona hidrológica de la cuenca del río Bogotá, junto con otras zonas densamente pobladas como Cali o Cartagena, muestran el índice de vulnerabilidad al desabastecimiento en la categoría de muy alto, es decir, son zonas con muy altas presiones por uso con respecto a la oferta disponible y una capacidad de regulación y retención muy baja.

La región hídrica de Bogotá es un concepto que ha venido estructurando la Empresa de Acueducto y Alcantarillado de Bogotá de la mano de institutos de investigación como el instituto Quinaxi liderado por el Doctor Ernesto Guhl. Es un área que se extiende unos $9.645 \mathrm{Km}^{2}$ e incluye la cuenca del río Bogotá, contados los 53 municipios de la cuenca y el distrito capital (Guhl-Nanneti, 2013).

La región se divide en tres subcuencas: Alta, media y Baja. La cuenca alta que arranca desde el nacimiento del río Bogotá y va hasta la frontera con el Distrito Capital, se caracteriza por tener una baja densidad poblacional (a pesar de que abarca 25 municipios y 49,1\% del área total) y vincularse con el recurso hídrico aportando funciones de protección, producción, regulación del agua. La cuenca media se compone de 11 municipios más Bogotá y concentra el 90\% de la población de la región, habitando el 32,5\% del área de la región hídrica. Se vincula con el agua principalmente por el consumo urbano y la generación de altas cargas contaminantes. Finalmente está la cuenca baja que incluye 17 municipios, representa el 18\% del área y se relaciona con el agua de la zona, porque allí se dan procesos de generación de energía, consumo turístico y es la parte receptora de alta contaminación proveniente de la cuenca media. 


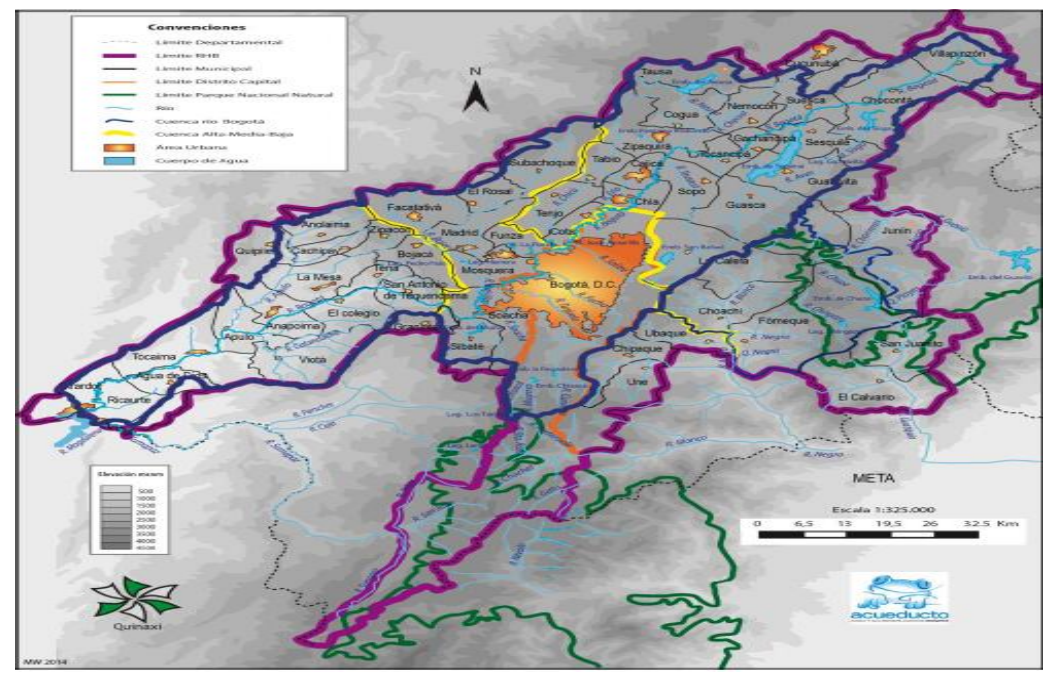

Figura 1Mapa de la región hídrica Bogotá. Fuente: (Guhl-Nanneti, 2013, pág. 13)

La región hídrica está delimitada por la línea morada, la línea verde encierra áreas de parques naturales, la línea azul incluye la cuenca de río.

La forma en que en la región funciona la relación producción - demanda de agua se puede esquematizar en los siguientes términos.

Podemos definir un conjunto de recursos hídricos compuestos por el sistema hídrico de la cuenca del río Bogotá con sus afluentes de aguas superficiales y subterráneas. Ellos, enmarcados en las condiciones climatológicas (Buytaert W. \&., 2012) y la preservación de los ecosistemas (Instituto Alexander von Humboldt, 2016) y el trasvase de cuenca a razón de 440,3 Mm³ (IDEAM, 2015), (Guhl \& Bernal, 2017) proveniente del río Guatiquía en la cuenca del Orinoco componen el stock del recurso disponible para el consumo en la región. Por otro lado, se encuentra la demanda de agua que se da en las nueve categorías referidas arriba (agropecuario, industrial, domiciliario, etc).

La demanda se ve influida por diferentes factores que moldean su comportamiento. En primer lugar, la variación de la población en general exhibe un comportamiento creciente y por tanto disminuye el stock del recurso. En segundo lugar, hay un marco institucional y una política nacional del recurso hídrico que también regulan el uso del agua. El primer factor se compone a su vez de una matriz legal que influyen en otros aspectos (Blanco, 2008) (Instituto para el desarrollo sostenible Quinaxi, 2014) en el control del agua, la planificación y participación. El segundo factor, la política nacional, está vigente desde 2010 y está orientada a que en el país se haga una Gestión Integral del Recurso Hídrico- GIRH- (en apartes siguientes de este documento se explica qué significa ese enfoque de gestión).

En tercer lugar, influye sobre el consumo el precio del servicio de acueducto y la venta de agua en bloque. El precio del servicio se refiere a la tarifa que se paga en cada municipio incluidas las tasas retributivas por uso y vertimiento y la venta de agua en bloque. 
Entre el stock de agua y la demanda hay un sistema de distribución para Bogotá y los municipios de la cuenca $(\approx$ 9.000.000 personas) que funciona conectando un sistema de reservas: Sistema agregado norte, Sistema agregado sur y sistema Chingaza que atienden una demanda de 15,44 m³/segundo (Jiménez-Aldana \& SantanaLópez, 2017); (Jiménez- Aldana, 2017 ). Así mismo, existe un sistema de plantas de tratamiento de aguas: Wiesner ubicada el nor-oriente de la ciudad en el municipio de la Calera, Tibitoc al norte, en el municipio de Tocancipá y Planta el Dorado ubicado al sur por la salida a la Ciudad de Villavicencio. El sistema en su conjunto en la actualidad genera un flujo de agua tratada de $15,73 \mathrm{~m}^{3} /$ segundo, aunque su capacidad instalada es de $27,3 \mathrm{~m}^{3} / \mathrm{segundo}$ (Jiménez-Aldana \& Santana-López, 2017).

Desafortunadamente el sistema de tratamiento de Bogotá tiene un alcance parcial en tanto no trata todas las aguas servidas de la misma ciudad y menos del resto de la región hídrica aunque hay que decir que a la fecha ya se cuenta con un convenio entre el Gobierno Nacional y la Alcaldía Mayor para culminar el proceso de descontaminación del río Bogotá (Espectador, 2017)

Finalmente, dentro de la descripción de los componentes dentro del esquema de oferta demanda en la región hídrica de Bogotá se encuentran la población rural y el sector empresarial ubicados cerca de las fuentes hídricas y que constituyen actores clave influyen directamente en el estado de los ecosistemas por donde corre el agua de la región. Por un lado, la población rural tiene la opción de actuar colectivamente para usar y conservar el recurso bajo la figura de asociación de usuarios de agua (AUA). En general, son organizaciones informales que cobran una pequeña cuota de membresía que utilizan para hacer mantenimiento a los tanques de almacenamiento y la tubería. Las AUA capturan el agua, la almacenan y la distribuyen desde pequeños arroyos. Estas asociaciones, en el caso colombiano, proveen agua al 41\% de la población rural (Murtinho, Tague, de Bievre, Eakin, \& Lopez-Carr, 2013).

Por otro lado, el sector empresarial articulado con el sector público mediante la figura de los fondos de agua. Esta alianza público-privada es una herramienta financiera a través de la cual los usuarios del agua compensan a los propietarios y a áreas protegidas por conservar bosques, páramos y suelos que proporcionan agua a la ciudad. El fondo de agua para Bogotá se llama Agua Somos y funciona desde 2008 (Agua Somos, 2017).

La oferta de agua de la región está compuesta por el sistema hídrico de la cuenca del río Bogotá con sus afluentes de aguas superficiales y subterráneas. Ellos, enmarcados en las condiciones climatológicas (Buytaert W. \&., 2012) y la preservación de los ecosistemas (Pérez-Hernández, 1998) (Instituto Alexander von Humboldt, 2016) y el trasvase de cuenca (IDEAM, 2015), (Guhl \& Bernal, 2017) proveniente del río Guatiquía en la cuenca del Orinoco componen el Stock del recurso disponible de $2241 \mathrm{Mm} 3$ / año (Huitaca consorcio - CAR, 2017). Por otro lado, se encuentra la demanda de agua (agropecuario, industrial, domiciliario, hidro-eléctrico) que consume $1866 \mathrm{Mm} 3$ (Huitaca consorcio - CAR, 2017).

No obstante, el margen de suficiencia a primera vista parece adecuado, el riesgo de desabastecimiento se empieza a generar por problemas en la gestión. En lo que respecta a la cuenca del río Bogotá se han identificado los siguientes: 
A) Desde la gestión del territorio - urbanismo

El factor común en los trabajos revisados es una inadecuada gestión del territorio. Si bien el patrón dominante de crecimiento urbano en Bogotá un desarrollo compacto de alta densidad (crecimiento poblacional significativo pero sin ampliar mucho el área urbana) (Parés-Ramos, Álvarez-Berríos, \& Mitchell-Aide, 2013), la inadecuada gestión del territorio se expresa en un crecimiento urbano sin planificación de largo plazo (Ricardo, 2014), conurbación desordenada (Osorio, 2008), (Motta, 2009) (Gaitan, 2010) presión por expansión urbana (Instituto Alexander von Humboldt, 2016), soluciones de vivienda en zonas de riesgo de inundación (Rojas, De Meulder, \& Shannon, 2015), destrucción de ecosistemas (Pérez-Hernández, 1998), la pérdida de páramos por la ampliación de la frontera agropecuaria (Cárdenas Agudelo, 2013) y una débil respuesta de protección (Blanco, 2008).

B) Desde el diseño institucional y los instrumentos de gestión del recurso. En este frente los autores han resaltado los siguientes problemas:

i. El diseño institucional no funciona como un sistema completo y coordinado en orden a encausar el uso y conservación del recurso en perspectiva de largo plazo. Por ejemplo: a) Los Planes de Ordenamiento y Manejo de Cuencas Hidrográficas (POMCA) son descriptivos y no sirve de base para establecer restricciones al uso de la tierra, b) la planeación de la cuenca no afecta la implementación de instrumentos económicos y administrativos para la gestión, c) hay confusión entre las instituciones ambientales y municipales sobre el alcance y relación entre POMCA y los Planes de Ordenamiento Ambiental (POT).

ii. Se observa una asignación ineficiente del agua en la región. Esto de se debe según Uribe (2005-6) a: a) procedimientos de concesiones centralizado largo y costoso para los pequeños usuarios de agua, b) la información relativa a la disponibilidad de agua y a la demanda en pequeñas subcuencas es incompleta, c) los procesos de sanción al uso ilegal de agua es centralizado, largo y complejo, d) la forma como se han reglamentado las tarifas por el uso de agua ha eliminado en gran parte de su potencial como instrumento económico y financiero de gestión ambiental

C) La cuenca del río Bogotá ya experimenta cambio y variabilidad climática en los siguientes términos (IDEAM; PNUD; Alcaldía de Bogotá; Gobernación de Cundinamarca; CAR; Corpoguavio; Instituto Alexander von Humboldt; Parques Nacionales Naturales de Colombia; MADS; DNP, 2014, pág. 8):

En general, en la Región Bogotá Cundinamarca la variabilidad climática interanual ha venido ocasionando aumentos en la temperatura de hasta $0.5^{\circ} \mathrm{C}$ y disminuciones en la precipitación de hasta $60 \%$ durante $\mathrm{El}$ Niño y disminuciones en la temperatura de hasta $0.5^{\circ} \mathrm{C}$ y aumentos en la precipitación de hasta $60 \%$ durante La Niña" “Adicionalmente, el análisis de los patrones climáticos en periodos de 30 años o más, muestran indicios de cambio climático en la región, los cuales se pueden llegar a expresar para el periodo 2041-2070, en aumentos promedio de $2^{\circ} \mathrm{C}$, e incremento promedio de la precipitación del orden de 1 a 2 $\mathrm{mm}$ más diariamente, en comparación a lo actual sobre el centro y occidente del departamento; y disminuciones promedio de entre 2 a $5 \mathrm{~mm}$ menos en comparación a lo actual, en el oriente de Cundinamarca"

Este es un factor que influye en que se califique de muy alto el Índice de Vulnerabilidad de Durante los últimos 20 años (IDEAM, 2019),y que en el POMCA del río Bogotá se ponga de manifiesto (Huitaca 
consorcio - CAR, 2017, pág. 468): "la necesidad de efectuar una muy cuidadosa conservación y administración del recurso hídrico para evitar posibles desabastecimientos en el futuro.

\section{MODELO Y RESULTADOS}

El sistema de abastecimiento de agua en la región hídrica de Bogotá tiene como centro de gravedad la relación entre reservas y consumo de agua. En tanto el margen de holgura de la oferta en relación con la demanda se reduzca significativamente, se activan las políticas de gestión del recurso como por ejemplo la ampliación de la oferta por medio de un ciclo de balance en el que al reducirse el margen del sistema se aprueban nuevos proyectos de ampliación de capacidad, los cuales llegan a concretarse con un regazo de entre 5 y 9 años pero que al final aumentan la capacidad de suministro y vuelven a aliviar la presión sobre el margen. Así mimo, cuando el margen se reduce, se activan las políticas de demanda que pueden observarse en el aumento de las tarifas o la limitación a los permisos de captación que permite reducir el consumo, con ello aumentar el nivel de agua disponible y disminuir la presión sobre el margen.

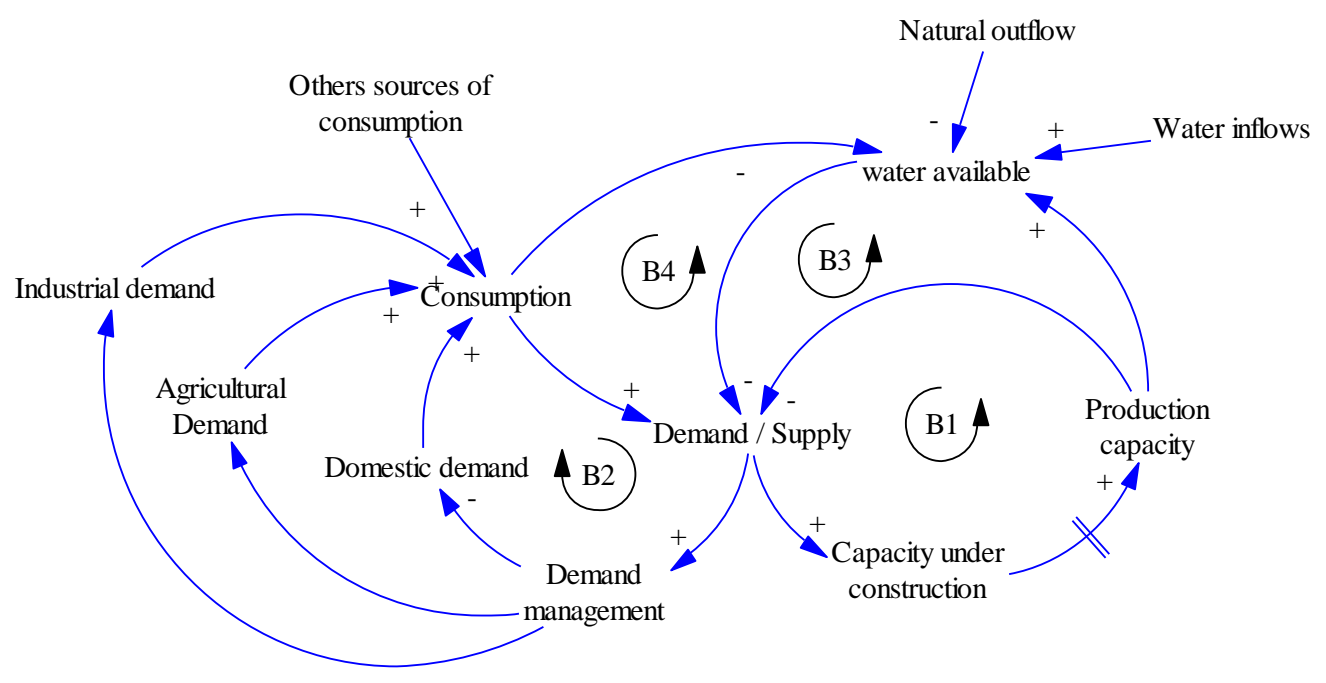

Figura 2. Hipótesis dinámica. Elaboración propia

Este artículo discute el posible desabastecimiento de agua para los habitantes y empresas de la cuenca del río Bogotá desde la Dinámica de Sistema, el cual es un tópico no estudiado hasta el momento.

La figura 2 presenta los componentes principales del modelo de dinámica de sistemas que se ha construido. Con este modelo se analizará el efecto de diferentes políticas hídricas sobre la administración de la cuenca. El período de simulación cubre los próximos 32 años. 


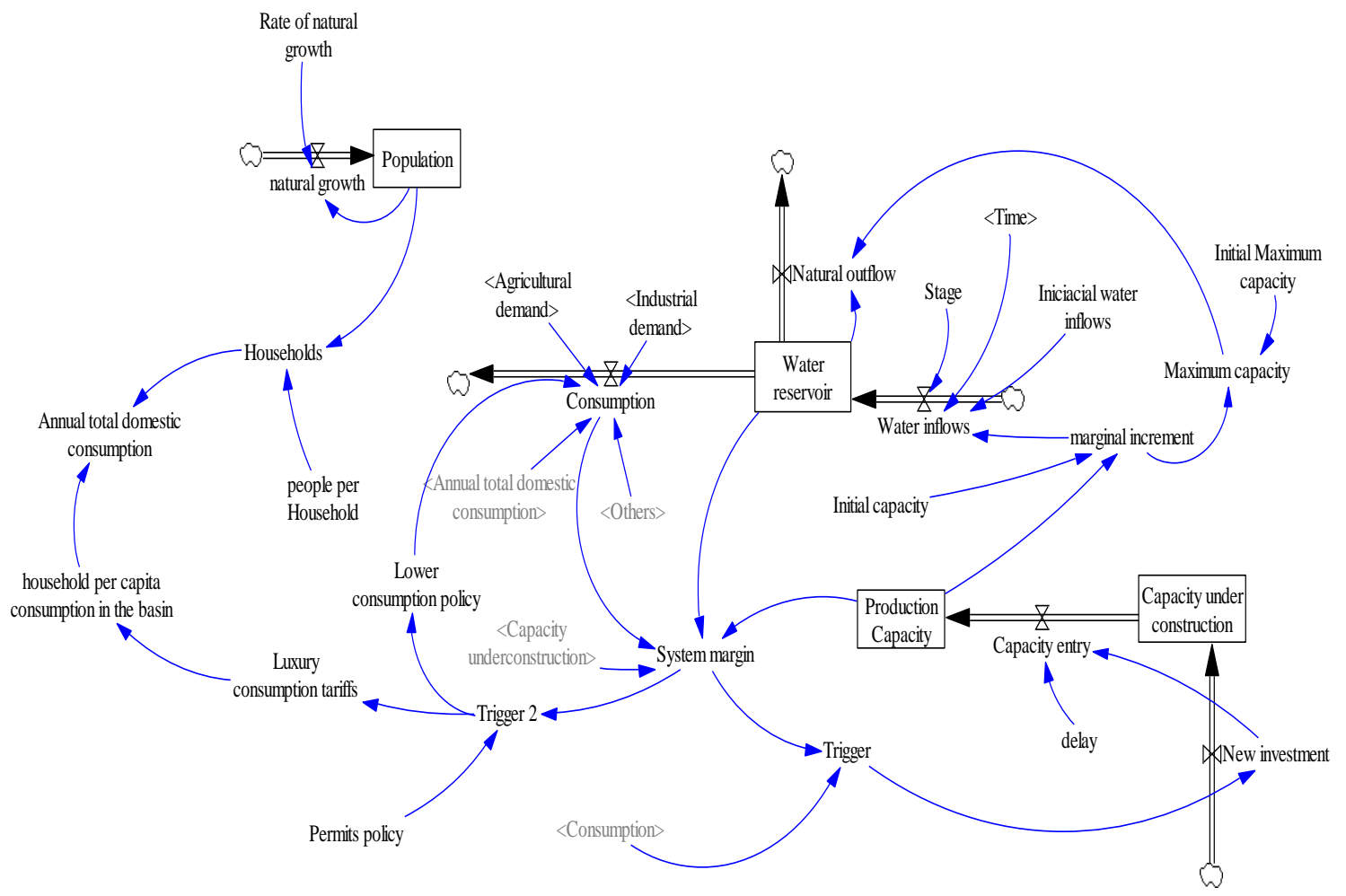

Figura 2. Diagrama de flujos y niveles para el sistema de abastecimiento de la región hídrica de Bogotá. Fuente: elaboración propia

La dinámica del estrés hídrico (demanda / oferta) y los dispositivos de política pública que interactúan con el estrés, se describen en un diagrama de flujos y niveles. El margen del sistema se define como proporción entre la diferencia de Demanda y oferta y la oferta. El margen puede oscilar entre o y 1 y su variabilidad depende cambios en la oferta del recurso como por ejemplo ampliación de las fuentes de agua Nuevos embalses, trasvases de cuenca, etc) y ampliación de la capacidad de producción entendida como más o mejores plantas de tratamiento. Así mismo pueden darse variaciones negativas en la oferta por presencia de sequías prolongadas, disminución en la producción de agua en páramos o en los afluentes superficiales. Del otro lado, el consumo total representa la agregación de cuatro tipos de demanda de agua en la región: El doméstico, industrial, agrícola y otros (que incluye la demanda pecuaria, la generación de energía por hidroeléctricas, entre otros.

Los dispositivos de política pública hídrica se activan cuando el margen del sistema llega al nivel de 20\%. En ese punto empieza el proceso de ampliación de la capacidad de suministro (lado de la oferta), bien por la adición de nuevas fuentes, bien por la ampliación o mejora de plantas de tratamiento. Este proceso toma varios periodos (entre 5 y 9 años) antes que esté en pleno funcionamiento. Así mismo, la política de demanda tiene como instrumentos básicos las tarifas (precios) y las concesiones (cantidades)

La tabla 1 muestra las principales ecuaciones del modelo y sus correspondientes unidades. Las ecuaciones reproducen la dinámica del margen del sistema y el impacto de la política pública hídrica para regular el suministro. 


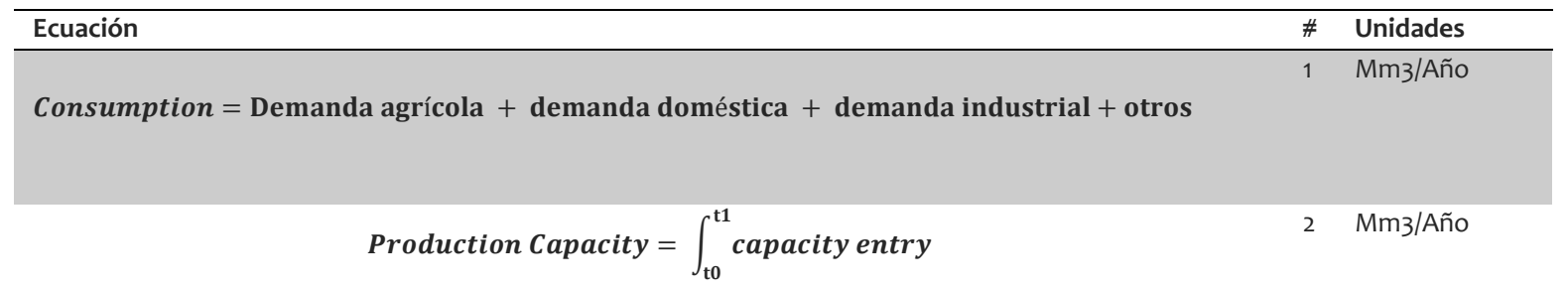

\begin{tabular}{|c|c|}
\hline System margin $=\frac{\text { Production capacity }- \text { Consumption }}{\text { Consumption }}$ & 3 \\
\hline $\begin{array}{l}\text { Trigger (disparador): es la señal del modelo para que la autoridad ambiental haga inversiones en } \\
\text { ampliación del suministro. Se activa cuando el margen del sistema es inferior a } 5 \%\end{array}$ & 4 \\
\hline $\begin{array}{l}\text { Trigger 2: es la señal del modelo para que la autoridad active una política de gestión de la demanda a } \\
\text { través de mecanismos que reduzcan el consumo. Ellos pueden ser restricciones al volumen vía limitación } \\
\text { de las concesiones de captación (permits policy) o pueden ser medidas de ajuste de la tarifa de consumo } \\
\text { suntuario (luxury consumption tariffs) Trigger } 2 \text { = IF THEN ELSE(System margin }< \\
0.055 \text {, Permits policy, 1) }\end{array}$ & 5 \\
\hline
\end{tabular}

\section{RESULTADOS}

Esta sección discute la corrida de simulación en referencia al tema del proyecto: determinar las condiciones para garantizar que el suministro de agua en la región sea sostenible en el largo plazo. En primera instancia, el diagrama de flujos y niveles presentado en la figura 3 hace una representación simplificada del sistema de oferta, demanda y gestión del recurso hídrico para el área de estudio. La simulación se ejecutó para una ventana de tiempo de 32 años (2018 - 2050) enmarcado en los supuestos indicados en la sección anterior.

En un primer escenario, se observa que la relación dinámica entre consumo (la suma del consumo industrial, agropecuario y domiciliario) y la oferta (capacidad de producción) no presenta inminencia de desabastecimiento en razón a que el gestor del recurso en la cuenca (CAR) realiza la aplicación de políticas combinadas: por un lado, planea y realiza una ampliación de la capacidad cuando observa que el margen del sistema se estrecha. La evidencia surgida del caso de Bogotá muestra que hay un rezago de entre 5 y 9 años en la puesta en funcionamiento de las nuevas inversiones. Por otro lado, al observar que el margen se reduce pone en marcha una política de demanda mientras que la demanda sigue una ruta de crecimiento sostenido (ver figura 3 )

Sin embargo, haciendo un ejercicio de incorporar la posibilidad de que ocurriera un año seco con lo cual la oferta disminuye y omitiendo el hecho de que la demanda aumenta, la situación en que hubiera desabastecimiento cada que tiene lugar dichos años (ver figura 4). Esto puede ocurrir porque la ampliación de la oferta no cambia su velocidad de ejecución con lo cual el desabastecimiento tendría lugar. Por supuesto ese ejercicio no considera la posibilidad de ampliar el trasvase de cuencas y un aumento en la severidad de las políticas de demanda que restrinjan el consumo industrial e inclusive agropecuario ni tampoco puede por ahora considerar la tensión que habría en un año seco con el sector hidroeléctrico. 


\section{Selected Variables}

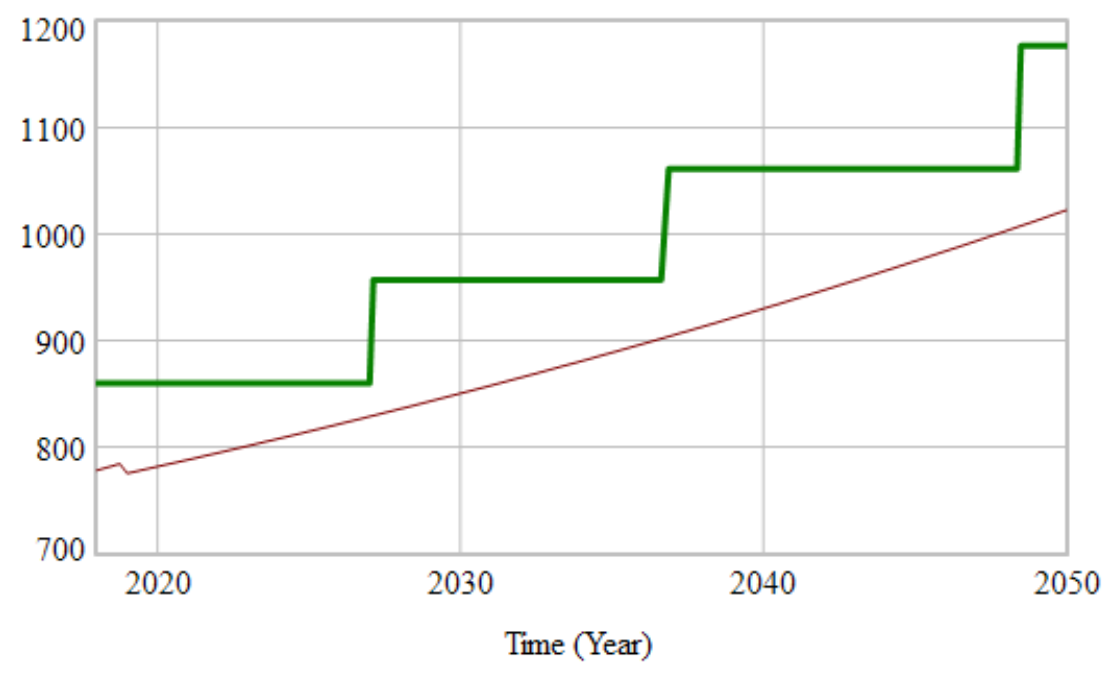

\section{『 Consuptiom : Current}

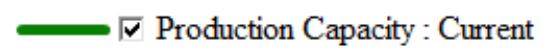

Figura 3. Comportamiento en el tiempo del Consumo y la oferta de agua en la cuenca del Río Bogotá. Fuente: Elaboración propia.

\section{Conclusiones}

Los resultados del trabajo de modelación del sistema de abastecimiento son consistentes con el comportamiento de las predicciones de los ejercicios proyección de la demanda y oferta de la Empresa de Acueducto y Alcantarillado de Bogotá (EAAB). En efecto, la citada empresa plantea la proyección de la demanda y la planeación de obras de ampliación de la oferta para la ciudad como se ve en la figura 4. En cualquier caso, es preciso aclarar que la gráfica se refiere al consumo de la ciudad de Bogotá no ha toda la cuenca y que la infraestructura planeada se hace realidad según lo previsto.

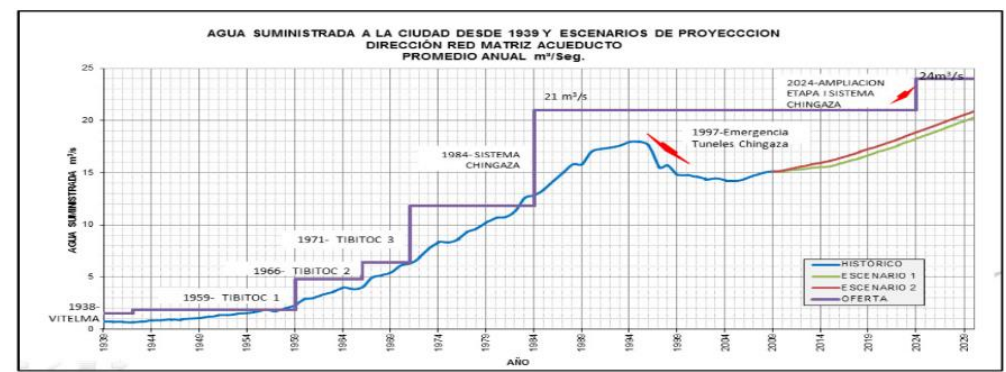

Figura 4 Oferta de agua en la ciudad de Bogotá y escenarios propuestos. Fuente: (Jiménez-Aldana, 2017, pág 665)

En un primer escenario, se observa que la relación dinámica entre consumo y la oferta no presenta inminencia de desabastecimiento en virtud a que el modelo por ahora no incorpora consideraciones que podrían estresar el sistema ostensiblemente: ¿cuál sería el impacto del cambio y la variabilidad climática sobre la principal fuente generadora de agua que son los páramos y sobre la demanda especialmente la agropecuaria? ¿Cómo se afecta el abastecimiento si se afecta el trasvase de agua de la fuente habitual si llega a ocurrir una disminución del caudal? 
Referencias bibliográficas

Agua Somos. (1 de mayo de 2017). Agua Somos, Bogotá- Colombia 2008. Obtenido de Fondos de agua: http://fondosdeagua.org/esp/agua-somos-bogota-colombia/

Blanco, J. (2008). Integrated Water resource Management in Colombia: Paralysis by analysis. International journal of water resources development 24 (1), 91-101.

Buytaert, W. \&. (2012). Water for cities: The impact of climate change and demographic growth in the tropical Andes. Water Resources Research 48 (8).

Buytaert, W., \& De Bièvre, B. (2012). Water for cities: The impact of climate change and demographic growth in the tropical Andes. WATER RESOURCES RESEARCH, VOL. 48, 1-13.

Cárdenas Agudelo, M. (2013). La gestión de ecosistemas estratégicos proveedores de agua. El caso de las cuencas que abastecen a Medellín y Bogotá en Colombia. Gestión y Ambiente, vol. 16 (1), 109-121.

Costa, D., Burlando, P., \& Priadi, C. (2016). The importance of integrated solutions to flooding and water quality problems in the tropical megacity of Jakarta. Sustianable Cities and Society (20), 199-209.

DANE. (29 de 06 de 2017). Producto Interno Bruto departamental 2005 - 2017 Base 2015. Obtenido de https://view.officeapps.live.com/op/view.aspx?src=http://www.dane.gov.co/files/investigaciones/pib/de partamentales/B_2015/PIB_Departamentos_2017preliminar.xls

DANE. (05 de 02 de 2020). Censo Nacional de Población y vivienda 2018-Colombia. Obtenido de ¿Cúantos somos?: https://sitios.dane.gov.co/cnpv/\#!/

Díaz, C. (2011). METABOLISMO DE LA CIUDAD DE BOGOTÁ D.C.: UNA HERRAMIENTA PARA EL ANÁLISIS DE LA SOSTENIBILIDAD AMBIENTAL URBANA. Bogotá: Tesis elaborada como requisito académico para optar al título de: Magister en Medio Ambiente y Desarrollo.

Espectador, E. (6 de octubre de 2017). Un salvavidas para el río Bogotá. El Espectador, págs. disponible en: https://www.elespectador.com/opinion/editorial/un-salvavidas-para-el-rio-bogota-articulo-716892.

Findeter, Financiera del desarrollo. (Agosto de 2017). Informe sectorial Agua potable y saneamiento básico Agosto 2017. Obtenido de https://www.findeter.gov.co/descargar.php?idFile=253376

FNA; FESCOL; Caracol Tv; CCB. (2015). Escenarios de futuro para la sostenibilidad ambiental de la región Bogotá Cundinamarca al 2025. Bogotá: Cámara de Comercio de Bogotá CCB.

Gaitan, J. (2010). Intervención alcalde de Chía en el foro Gobernabilidad Regional: Los municipios y Bogotá. Debates de Gobierno Urbano \# 4. Instituto de Estudios Urbanos / Universidad Nacionalde Colombia, (pág. 7). Bogotá.

GONZÁLEZ-MORENO, L. V., ARAGÓN-PINZÓN, A. M., \& MORENO-GARCÍA, R. (2015). DETERMINAR LA VULNERABILIDAD AL DESABASTECIMIENTO HÍDRICO DEL PARAMO GUERRERO Y ESTABLECER LAS POSIBLES MEDIDAS DE ADAPTACION Y MITIGACIÓN. Bogotá: Tesis de especialización en recursos hídricos. 
Guhl, E., \& Bernal, P. A. (2 de julio de 2017). Blu Verde. Debate: ¿Sabana de Bogotá tiene recursos hídricos para satisfacer demanda? Obtenido de Blu Radio: https://www.bluradio.com/medio-ambiente/debatesabana-de-bogota-tiene-recursos-hidricos-para-satisfacer-demanda-145878

Guhl-Nanneti, E. (2013). LA REGIÓN HÍDRICA DE BOGOTÁ. Revista Academia Colombiana de Ciencias Vol 37 (144) septiembre, 327-341.

HOFSTE, R. W., KUZMA, S., WALKER, S., SUTANUDJAJA, E. H., BIERKENS, M. F., KUIJPER, M. J., . . GALVIS, S. (2019). AQUEDUCT 3.0: UPDATED DECISION-RELEVANT GLOBAL WATER RISK INDICATORS. Washington: World Resources Institute.

Hossain, N., \& Bahauddin, K. (2013). Integrated water resource management: a case study of Dhaka city, Bangladesh. Journal of water and land development 19, $39-45$.

Huitaca consorcio - CAR. (2017). AJUSTE DEL PLAN DE ORDENACIÓN Y MANEJO DE LA CUENCA DEL RÍO BOGOTÁ. Bogotá: CAR.

IDEAM. (2015). Estudio Nacional del Agua 2014. Bogotá: IDEAM.

IDEAM. (2015). Estudio Nacional del Agua 2014. Bogotá, D.C.

IDEAM. (2019). Estudio Nacional del Agua 2018. Bogotá: Ideam.

IDEAM. (2019). Estudio Nacional del Agua 2018. Bogotá, D.C.

IDEAM; PNUD; Alcaldía de Bogotá; Gobernación de Cundinamarca; CAR; Corpoguavio; Instituto Alexander von Humboldt; Parques Nacionales Naturales de Colombia; MADS; DNP. (2014). Estrategia Regional de Adaptación al Cambio Climático para la Región Bogotá Cundinamarca :Portafolio De Perfiles De Proyectos Priorizados Para La Mitigación Y La Adaptación Al Cambio Climático En La Región Bogotá Cundinamarca. PRICC. Bogotá: IDEAM.

Instituto Alexander von Humboldt. (2016). Recomendación para la delimitación, por parte del Ministerio de Ambiente y Desarrollo Sostenible, del Complejo: Complejo de Páramos de Cruz Verde-Sumapaz / de Páramos de Chingaza a escala 1:25.000. Bogotá: Insituto de investigación de recursos biológios Alexander von Humbolt y Fondo de Adaptación.

Instituto para el desarrollo sostenible Quinaxi. (2014). diseño institucional para aplicar la gestión integrada del agua y del territorio GIAT informe final. Bogotá.

Ivanova, Y. (2013). EVALUACIÓN DE LA HUELLA HÍDRICA DE LA CIUDAD DE BOGOTÁ COMO UNA HERRAMIENTA DE GESTIÓN DEL RECURSO HÍDRICO EN EL ÁREA URBANA. Bogotá: Tesis de Maestría en Gestión Ambiental.

Jiménez- Aldana, M. (2017 ). Integral Network Management: A Case Study of Bogotá and the Empresa de Acueducto, Alcantarillado y Aseo de Bogotá, EAB ESP. Procedia Engineering 186, 654 - 665.

Jiménez-Aldana, M., \& Santana-López, F. (2017). Water Distribution System of Bogotá city and its surrounding area, Empresa de Acueducto y Alcantarillado de Bogotá - EAB E.S.P. Procedia Engineering 186, 643-653.

Martín, M. (6 de mayo de 2015). São Paulo teme el caos social por la gravedad de la sequía. Los militares debaten junto a académicos y autoridades cómo solucionar el problema del desabastecimiento. EL PAïS, pág. https://elpais.com/internacional/2015/05/06/actualidad/1430927886_428471.html. 
Motta, R. (2009). PRIORIDADES AMBIENTALES EN LA REGIÓN CENTRAL DE COLOMBIA Y SU PROBLEMÁTICA EN EL RECURSO HÍDRICO. Revista Republicana \# 6, 99-110.

Murtinho, F., Tague, C., de Bievre, B., Eakin, H., \& Lopez-Carr, D. (2013). Water Scarcity in the Andes: A Comparison of Local Perceptions and Observed Climate, Land Use and Socioeconomic Changes. Human Ecology 41, 667-681.

Osorio, J. (2008). LA HISTORIA DEL AGUA EN BOGOTÁ: UNA EXPLORACIÓN BIBLIOGRÁFICA SOBRE LA CUENCA DEL RÍO TUNJUELO, EN EL SIGLO XX. Memoria y sociedad 12 (25) julio diciembre, 107-116.

Parés-Ramos, I. K., Álvarez-Berríos, N. L., \& Mitchell-Aide, T. (2013). Mapping Urbanization Dynamics in Major Cities of Colombia, Ecuador, Perú, and Bolivia Using Night-Time Satellite Imagery. Land \#2, 37-59.

Pérez-Hernández, E. (1998). La crisis del agua en Bogotá. Bitácora Urbano Regional. Universidad Nacional de Colombia Facultad de Artes, Departamento de Urbanismo, 55-59.

Pérez-Preciado, A. (2000). El problema del Río Bogotá. En A. Pérez-Preciado, Bogotá y Cundinamarca : expansión urbana y sostenibilidad (págs. 21-60). Bogotá.

Räsänen, A., Juhola, S., Monge, A., Käkönen, M., Kanninen, M., \& Nygren, A. (2017). Identifying mismatches between institutional perceptions of water-related risk drivers and water management strategies in three river basin areas. Journal of Hydrology 550 , 704-715.

Redacción BBC Mundo. (19 de 01 de 2018). Ciudad del Cabo: el "día cero" en el que por primera vez una gran ciudad del mundo podría quedarse sin agua. BBC, págs. http://www.bbc.com/mundo/noticiasinternacional-42742476.

Ricardo, L. (2014). El papel del agua en una ciudad como Bogotá. revista Ciudades, Estados y política 1(2), 51-60.

Rojas, C., De Meulder, B., \& Shannon, K. (2015). Habitat International 48 , 177-187.

Rudas, G. (2012). Inversiones obligatorias en conservacion y manejo de cuencas hidrográficas. Economía Colombiana. Revista De La Contraloría General De La República 334, 100-112.

Silva, N., \& Plascencia, Á. (22 de marzo de 2016). Dos megaciudades, una crisis. Las dos metrópolis más grandes de América Latina afrontan el reto de gestionar, en una década, un sistema sustentable de agua. EL PAïs, pág. https://elpais.com/internacional/2016/03/21/actualidad/1458597371_473379.html.

Uribe, E. (2005-6). The allocation of water resources in the Bogotá Savanna region: case study. Documento CEDE, $1-30$.

van den Brandeler, F., Gupta, J., \& Hordijk, M. (2018). Megacities and rivers: Scalar mismatches between urban water management and river basin management. Journal of Hydrology https://doi.org/10.1016/j.jhydrol.2018.01.001.

Varis, O., Biswas, A. K., Tortajada, C., \& Lundqvist, J. (2006). Megacities and water management. International journal of water resources development 22 (2), 377 - 394. 